$01 / 14 / 19$

\title{
NON-SYNAPTIC TRANSMISSION AND THE FOUNDATIONS OF
}

\begin{abstract}
AFFECT
Gil B. Carvalho and Antonio Damasio*

Brain and Creativity Institute, Dornsife College of Letters, Arts, and Sciences University of Southern California, Los Angeles

*Correspondence: damasio@usc.edu
\end{abstract}

Keywords: Non-synaptic transmission, synapse, neurotransmitters, interoceptive nervous system, volume transmission, paracrine, myelin, blood-brain barrier, interoception, affect

Supported by The Mathers Foundation and the Berggruen Institute

\begin{abstract}
Neuroscience has overwhelmingly and understandably focused on the synaptic modality of signal transmission. There is evidence, however, that from an evolutionary perspective, non-synaptic transmission (NST) preceded synaptic signaling. Moreover, in modern nervous systems, NST coexists and extensively interacts with synaptic transmission modifying neuronal dynamics. In fact, NST remains widespread in complex animals, especially within the interoceptive system where the dearth of insulating barriers such as myelin sheaths and the blood-brain barrier enhances the communication between neural and non-neural tissues mediated by NST. We suggest that this physiological arrangement makes a fundamental contribution to interoception - the process of sensing visceral states — which is an essential underpinning of the capacity to feel and the foundation of affective processing.
\end{abstract}


01/14/19

\section{THE DAWN OF SIGNAL TRANSMISSION}

Synapses mediate and finely regulate nerve impulse conduction throughout the nervous system, playing a central role in physiological responses, movement and thought. Not surprisingly, synapses are commonly regarded as biological marvels and fundamental pillars of neural function. Nonetheless, several lines of evidence suggest that the intricately sophisticated synapse stems from comparatively humble beginnings, and that, no less importantly, exemplars of these earlier forms continue to play crucial roles in modern nervous systems. ${ }^{1}$

Signal transmission can be found in rudimentary and ancient organisms. In fact, most modern neurotransmitters arose and functioned as signaling agents long before the appearance of neurons in evolution. For example, in the sponges that make up the phylum Porifera, GABA, glutamate, acetylcholine and serotonin operate in paracrine ${ }^{*}$ mode, in the absence of synapses and neurons ${ }^{2}$. Genetic, anatomical and biochemical analyses indicate that the paracrine pathways of Porifera are evolutionarily related to certain synaptic elements present in metazoans, although it remains unclear whether sponges preceded the evolutionary appearance of neurons or underwent a loss of neural cell types ${ }^{2}$. The origin of the molecules commonly referred to as "neurotransmitters" dates even farther back than Porifera or even the animal kingdom. In fact, most of the main neurotransmitters found in the mammalian nervous system play a central role in organisms

\footnotetext{
*The term Paracrine designates signaling involving a molecule secreted by a cell and interacting with receptors on neighboring cells; the term Intracrine refers to intracellular signaling by a molecule acting in the cell where it is produced; Autocrine signaling involves a molecule secreted by a cell and interacting with receptors on that same cell; Endocrine signaling involves a molecule secreted by a cell and interacting with receptors on distant cells.
} 
01/14/19

devoid of nervous systems, such as unicellular eukaryotes and even bacteria ${ }^{3,4}$. Acetylcholine, for example, may date as far back as 3 billion years ${ }^{5}$ while animals are only thought to have arisen $\sim 600$ million years ago and nervous systems even later ${ }^{6}$. Neurotransmitters were not "neuro" for the vast majority of their existence.

One theory that has been advanced to account for the evolution of signal transmission proposes that metabolites initially functioned in an autocrine or intracrine manner, in bacteria. Later, in unicellular eukaryotes, they operated as messengers within and between unicellular organisms ${ }^{2,7-9}$. Even later, in multicellular organisms, these messengers continued to fulfill their inter-cellular function, but now within the perimeter of a single organism ${ }^{10,11}$. Thus in early animals inter-cellular communication was largely mediated by signals released into the extracellular matrix (i.e. paracrine signaling). During the development of Metazoa, evolutionary forces may have favored more direct and faster communication modalities, leading to changes in cellular morphology — the development of axons and dendrites — and to the compartmentalization of the neurotransmitter release channels. These changes would have facilitated a gradual transition from dispersed release to an open synaptic architecture, and, finally, to closed synapses, thus effecting a transition from paracrine to electrochemical signal transduction ${ }^{2}$. This conceivable evolutionary path would have substantially increased channel selectivity and speed in point-to-point transmission, an obvious advantage in the case of movement and, eventually, of perception and cognitive abilities such as memory and the manipulation of percepts, actual or recalled. In this perspective, synapses are an evolutionary specialization of nonsynaptic transmission (NST) offering refinement and efficiency. 
$01 / 14 / 19$

\section{NST IN MODERN NERVOUS SYSTEMS}

In spite of the advantages conferred by the synapse, the rudimentary NST precursor was not replaced and has remained prevalent. NST is prominent in numerous invertebrate phyla, including species where it coexists with synaptic signaling, and remains widespread in the vertebrate nervous system $^{11,12-14}$, where nonsynaptic receptors may actually outnumber synaptic ones ${ }^{15,16}$. In fact, most neurotransmitters can act nonsynaptically, and in many cases do so predominantly ${ }^{15,17,18}$. For example, a large fraction of acetylcholinergic, serotonergic and noradrenergic varicosities are nonsynaptic; in some areas of the nervous system, as many as 80$90 \%$ do not show the structural specializations that characterize synapses and are therefore thought to release the neurotransmitters for diffusion in the extracellular compartment, thereby activating more or less remote receptors ${ }^{19,20-23}$. This scenario is supported by fact that the monoaminergic (dopamine, noradrenaline and serotonin) fiber terminals from which the transmitters are released are often not adjacent to the respective receptors ${ }^{24-26}$, indicating that following their release the molecules must diffuse in the extracellular space. The numerous examples of monoamine involvement in NST include the vertebrate retina (where dopamine may play a role in light-dark adaptation ${ }^{28}$ ) and the amygdala ${ }^{27}$. Dopamine is also believed to operate non-synaptically in the spinal cord and in the cortical microvasculature where it helps regulate blood flow ${ }^{26,29,30}$. Even neurotransmitters typically associated with synaptic transmission, such as glutamate and GABA, have been shown to operate less conventionally and signal via extrasynaptic pathways, e.g. in the cerebellum and hippocampus $(34,35)$. 
01/14/19

The scope of NST extends far beyond canonical neurotransmitters. ATP and neuropeptides also have a well-established non-synaptic role $(31,32,33)$. Examples of peptide signaling include cytokines such as interleukin-1b(IL-1b) (42), and B-endorphin, which can be detected in cortical areas distant from the cell bodies where it originates and is thought to migrate long distances via diffusion in the extracellular fluid and cerebrospinal fluid (36). In fact, all hormones, peptide and otherwise, can be said to function via nonsynaptic signaling. Endocrine signaling, the diffusion of signaling molecules via the bloodstream, can be seen as a modality of NST.

ATP and neuropeptides also have a well-established role in NST ${ }^{31,32,33}$. Even neurotransmitters typically associated with canonical synaptic transmission, such as glutamate and GABA, have been shown to play less conventional roles and signal via extrasynaptic pathways, e.g. in the cerebellum and hippocampus ${ }^{34,35}$. Yet another example of a diffusing substance in the CNS is $\beta$-endorphin, which can be detected in cortical areas distant from the cell bodies where it originates; it is thought to migrate long distances via diffusion in the extracellular fluid and cerebrospinal fluid ${ }^{36}$.

Gases and ions can also mediate NST. For example, nitric oxide (NO) is a widespread paracrine transmitter (neuro- and otherwise) with well-established roles in a plethora of physiological and pathological phenomena such as vasodilation, gastro-intestinal smooth muscle relaxation, learning and memory, and the immune response ${ }^{37}$. Carbon monoxide $(\mathrm{CO})$ is another example of a gas involved in non-synaptic signaling. $\mathrm{CO}$ is produced in vascular smooth muscle cells and plays a role in the regulation of blood vessel tone. It may also function as a 
01/14/19

neurotransmitter ${ }^{38}$. NO and CO can cross cell membranes and diffuse away from their production sites, ultimately acting on cell surface and intracellular targets alike ${ }^{35}$. As for ions, extracellular potassium $\left(\mathrm{K}^{+}\right)$produced as a result of impulse transmission and synaptic currents can also modulate neuron signal transmission and glial function ${ }^{39}$. Additionally, protons $\left(\mathrm{H}^{+}\right)$ modulate membrane excitability, synaptic function and gap-junction conductance — for example, $\mathrm{H}^{+}$can bind NMDA receptors ${ }^{40}$. Finally, receptors for $\mathrm{Ca}^{2+}$ are present in the cell membranes of nerve terminals in several brain regions ${ }^{41}$.

NST is prevalent across most areas of the nervous system. In the CNS, instances of NST can be found at virtually every level of the neuraxis, especially in subcortical regions, namely the spinal cord, brain stem, basal ganglia and some hypothalamic nuclei. NST may play a particularly prominent role in limbic structures ${ }^{11}$. In the peripheral nervous system, chemosensitivity has been widely documented along the axonal trunks of peripheral sensory fibers and of the vagus nerve ${ }^{43-47}$, strongly pointing to a role for NST in interoception/visceroception. NST also seems widespread in peripheral ganglia. It can be observed in roughly $90 \%$ of spinal ganglion neurons ${ }^{48}$ and has also been reported in the nodose ganglion of the vagus ${ }^{49}$. Yet its physiological roles and mechanisms have remained unclear. NST in peripheral ganglia is thought to be neurotransmitter-mediated, although the specific transmitter in question has not been identified ${ }^{49-51}$. The extensive crosstalk observed among neighboring cell bodies elicits changes in membrane potential and firing threshold, which in turn control the likelihood of action potential propagation along sensory axons ${ }^{52}$. Thus DRG somas have the potential to act as sensory pathway gatekeepers and may operate as a synchronization mechanism. 
01/14/19

NST is also prevalent in the autonomic nervous system. Examples include 1) autonomic neuroeffector junctions ${ }^{53}$ and 2) the enteric nervous system, where noradrenaline released from sympathetic nerves acts on neighboring cholinergic terminals to prevent acetylcholine release, in spite of the fact that no synaptic contacts exist between the two types of fibers ${ }^{35}$.

Given its ubiquity in modern nervous systems, NST is increasingly recognized as a major modality of neurotransmission on a par with the canonic synaptic variety ${ }^{15}$.

Of note, the physiological role of NST extends far beyond the nervous system. Unlike synapses, which connect neurons to each other or to cells such as myocytes or glia, NST has virtually no cell type restriction. Examples of NST between non-neuronal cells abound ${ }^{26}$. Certain endocrine hormones, such as insulin or cortisol, are obvious examples. A paracrine role in the pancreas has also been described for glutamate, acetylcholine and GABA. The latter is secreted by pancreatic $\beta$-cells and binds membrane receptors on the surface of neighboring islet cells, possibly regulating insulin and glucagon secretion ${ }^{54,55}$. Acetylcholine is thought to be a nearly universal signaling molecule, playing a paracrine, non-neuronal role in the lung bronchial epithelium, in erythrocytes, in ovarian endocrine cells and the immune system, among others 5,56,57. These observations demonstrate that NST establishes communication pathways involving neuronal and non-neuronal cells alike. 
01/14/19

\section{THE DISTINCTIVE PHYSIOLOGY OF NST}

By comparison to synaptic transmission, NST presents a number of striking differences. NST entails an agent (neurotransmitter, neuropeptide, ion or gas) being released by a cell, diffusing across the extracellular space and exerting its effects by reaching one or more target cells where it can act by e.g. binding to extra-synaptic membrane receptors. However, unlike the case of synapses, where the point of communication is physically well defined, NST does not require terminal varicosities or boutons and the two or more cells involved in the communication need not be adjacent. The signaling agent diffuses three-dimensionally in the extracellular space, predominantly in the intercellular interstitium but in some instances also in the cerebrospinal fluid and even in the blood stream. Signals travel over variable distances as a consequence of energy gradients ${ }^{58,59}$. For example, the monoamines dopamine, noradrenaline and serotonin are prominent NST messengers involved mostly in short-range transmission, on the order of micrometers ${ }^{60}$. Dopamine gets released into the extracellular fluid, where its receptors are often located in the non-synaptic regions of dendrites, axons and cell somas ${ }^{61,62}$. These receptors have an effective radius of 2-8 $\mu \mathrm{m}^{63}$, i.e. 2-3 orders of magnitude larger than the typical synaptic cleft width of $\sim 12-20 \mathrm{~nm}^{64}$. In sharp contrast, neuropeptides (beta-endorphin, oxytocin, etc) are typical long-distance signalers, covering millimeters or even meters in the extreme cases of ghrelin, leptin and insulin, which are produced in the gastro-intestinal system and can act as far away as the CNS ${ }^{65}$.

NST is affected by the myriad physicochemical factors that have an impact on extracellular diffusion. These include electrical potentials and concentration, pressure, and temperature 
01/14/19

gradients ${ }^{66,67}$. Extasynaptic neurotransmitters are also more vulnerable to inactivating mechanisms such as degrading enzymes present in the extracellular fluid and plasma membrane transporters. The latter play an especially intriguing role, since in addition to their uptake of extracellular transmitters — which reduces the concentration of diffusing molecules - they can operate in "reverse mode" by releasing transmitters from the neuronal cytoplasm into the surrounding extracellular space and thereby enabling NST ${ }^{35}$. Together these elements determine the clearance and half-life of non-synaptic agents and therefore effectively and dynamically regulate NST. 
$01 / 14 / 19$

\section{THE FUNCTIONAL ADVANTAGES OF NST}

The widespread distribution of NST in the nervous system and its role in myriad biological processes, from synaptic plasticity to behavior, strongly indicate that NST is more than a mere evolutionary relic. Another powerful argument in favor of the physiological relevance of NST is its remarkable structural conservation. For example, the architecture of central monoaminergic neurons (with its neurotransmitter-releasing terminals typically distant from the receptor sites) is identical across several mammalian species, indicating functional relevance ${ }^{67}$.

The persistence of NST suggests a substantial evolutionary benefit. In fact, although at first glance it may appear metabolically wasteful compared to the targeted and geographically confined synapse, NST may provide advantages in specific circumstances. While synapses clearly are a more direct and faster way to transmit a signal between two specific cells i.e. "oneto-one" transmission, NST becomes energy-efficient in instances of "one-to-many" communication, in which one cell can potentially influence hundreds or thousands of others without the need for the elaborate wiring network of axons that synaptic signaling would require to achieve the same effect ${ }^{68,69}$. NST may also allow faster regulation ${ }^{70}$. For example, in a local context where one cell secretes a messenger, e.g. a neuropeptide, hundreds of neighbors are potentially exposed to it, but their receptivity to the signal is determined individually by the expression of the respective membrane receptor. An unresponsive subpopulation of cells can be rapidly turned "on" merely by upregulating the expression of one protein, the membrane receptor in question (Fig. 1). Conversely, switching the cell "off” requires only that the receptor be internalized or downregulated. In contrast, synaptic transmission requires entire axons/synapses 
01/14/19

to be built anew, a far more energy- and time-consuming process ${ }^{34}$. Thus NST may allow a population of cells exposed to one or many signals to regulate signal transmission quickly and with energetic efficiency ${ }^{71}$. Synapses and NST are thus likely to be complementary, contextdependent methods of signal transmission ${ }^{68}$. Synaptic signaling provided an evolutionary advantage in cases in which specific information is relayed between two well-defined points in a time-sensitive manner (e.g.movement, reflexes, perception), while its predecessor, NST, remained the approach of choice for instances of one-to-many transmission, with fewer time constraints. 
$01 / 14 / 19$

\section{SYNAPTIC AND NONSYNAPTIC TRANSMISSION: COMPLEMENTARY APPROACHES TOWARD A COMMON GOAL}

Synapses and NST coexist in an intricate interactive balance of extensive crosstalk and mutual regulation. How are these two signal transmission modes integrated and translated into neural function? What are the molecular mechanisms of their crosstalk? Transmembrane receptor heteromers appear to play a central role. Ion channels and G-protein coupled receptors (GPCRs) act as sensors of synaptic and non-synaptic signals, and exhibit direct, allosteric interactions which integrate distinct extracellular signals into common downstream molecular pathways $60,72,73$.

Another integration mechanism involves the diffusion of neurotransmitters from the synaptic compartment to the surrounding extracellular space. Leaked synaptic transmitters can (a) activate nonsynaptic membrane receptors, effectively merging synaptic transmission and NST, or (b) diffuse back into the synaptic compartment of neighboring synapses, a phenomenon known as intersynaptic “spillover" (Fig. 2). All different permutations of this scenario have been reported ${ }^{34}$. Whether of synaptic or nonsynaptic origin, neurotransmitters can affect membrane potential, thereby modulating the likelihood of action potential firing. In the context of the synapse these ionic changes are known as post-synaptic potentials (PSPs) and can be excitatory or inhibitory, depending on whether the post-synaptic membrane undergoes depolarization or hyperpolarization, respectively increasing or decreasing the likelihood of the neuron reaching threshold ${ }^{74}$. Nonsynaptic transmitters can have similar effects ${ }^{48}$. In fact, nonsynaptically generated PSPs can have exceptionally long durations ${ }^{35}$, probably due to the longer-lasting and 
01/14/19

slower nature of NST. Since such changes in membrane potential are graded and potentially additive, this represents a potential mechanism for synaptic/non-synaptic signal integration. Thus a given neuron's likelihood of firing is determined by the integration of the mechanisms acting upon it and those mechanisms are both synaptic and non-synaptic, both excitatory and inhibitory.

In addition to serving parallel and complementary functions, there is some evidence that ST and NST can, on occasion, fulfill overlapping roles. For instance, in Parkinson's disease, which is due to loss of dopaminergic neurons in the substantia nigra, one physiological compensatory strategy involves a potentiation of $\mathrm{NST}^{26}$. Moreover, the therapeutic effect of L-Dopa, the key treatment of Parkinsonism, is attributable to NST since L-Dopa is converted to dopamine by non-dopaminergic neurons and released non-synaptically, allowing it to reach its target cells by diffusion ${ }^{26,75}$. 
01/14/19

\section{THE BRAIN-BODY INTERFACE, NST AND THE PHYSIOLOGY OF AFFECT}

There is a striking affinity between NST and unmyelinated neuronal fibers. Unmyelinated axons were followed by an evolutionary specialization — the myelin sheath — that enhanced the speed of signal transmission, but as is the case with NST, unmyelinated fibers were not simply replaced by the more modern, faster variety. They remain widely represented in complex nervous systems, including those of humans. Myelination is a metabolically costly process but that fact alone is unlikely to explain the less than universal adoption of myelin ${ }^{76}$. The topographical overlap of NST and unmyelinated axons is another sign of their affinity. For example, unmyelinated, tightly packed fibers are more prone to ephaptic interactions, which depend on NST ${ }^{77}$. Moreover, unmyelinated axons of the peripheral nervous system are sensitive to extrasynaptic neurotransmitters such as acetylcholine, serotonin, norepinephrine and ATP, even if these molecules only reach the axonal trunk, away from the nerve terminals ${ }^{44-46,78}$. In the CNS, the subcortical nervous system is home to a substantial proportion of unmyelinated structures, and is also the origin of many nonsynaptic transmitters ${ }^{22}$. Specifically, medial structures of the neuraxis such as those in the limbic system and brain stem, which are prominently involved in the regulation of homeostasis and affect (drives, emotions, feelings), are especially rich in unmyelinated fibers and monoaminergic/peptidergic neurons and are thought to signal prominently via NST ${ }^{79,80}$. In contrast, the more lateral domains of the mammalian brain (including the main sensorymotor systems, the association cortices and the cerebellum) are especially rich in well myelinated axons ${ }^{11,81}$. Finally, the interoceptive nervous system — a key physiological contributor to the phenomena of feeling - exhibits a high concentration of unmyelinated fibers, as well as numerous instances of NST ${ }^{45,49,50,78}$. The structures where NST 
01/14/19

is prevalent tend to have abundant denuded axons, whereas structures relying mostly on synaptic communication-those involved in movement, cognition, sensory perception-appear to be predominantly served by myelinated fibers. This bimodal distribution matches the contrasting temporal scales of the related neural process. Myelination is an improvement that mainly serves time-sensitive synaptic transmission, whereas unmyelinated fibers and NST pertain largely to processes that can afford slower and less precise transmission, which is the case with certain aspects of interoceptive information and feelings ${ }^{76,82}$.

The conspicuous overlap between NST and unmyelinated fibers is likely to have functional relevance. In effect, myelin is a physical obstacle to NST, the myelin sheath acting as a potential block to both secretion and reception of the messenger ${ }^{83-85}$. Unmyelinated fibers are comparatively exposed and thus able to sense and emit circulating molecules, including neurotransmitters.

Another striking feature of the interoceptive nervous system are the localized gaps in the blood-brain barrier (BBB) — or blood-nerve barrier, as it is commonly designated in the peripheral nervous system. The BBB exhibits particularly conspicuous gaps in spinal ganglia. Each ganglion is divided into two regions, one housing most cell bodies and another containing mainly nerve fibers. The former is greatly enriched for vascularization, with a dense network of capillaries intimately wrapped around the sensory neuron somas. In contrast, the nerve fiber-rich area of the ganglion, as well as the adjacent nerves, are almost entirely devoid of blood vessels ${ }^{86}$. Moreover, the endothelium of the capillaries supplying the ganglia shows large fenestrations and is permeable to blood-borne agents of high molecular weight, properties reminiscent of areas 
01/14/19

of the CNS lacking a BBB, such as the area postrema, and also observable in autonomic sympathetic ganglia and the enteric nervous system ${ }^{87}$. In sharp contrast, the capillaries present within nerve trunks tend to be impermeable. Presumably as a result of this selective vulnerability, blood-borne toxins preferentially affect the sensory pathways, and indeed can accumulate in spinal ganglia, whereas motor pathways are comparatively spared ${ }^{86}$. This selectivity is revealed by intra-venous administration of labeled albumin, subsequently found to accumulate in the extravascular, peri-neuronal space of the cell body-rich, but not the nerve fiber-rich area of spinal ganglia. This strongly indicates that the dense network of capillaries enveloping the sensory cell bodies bear a specific and topographically conscribed gap in the $\mathrm{BBB}$, presumably a result of the epithelial fenestrations and molecular differences in tight junction composition ${ }^{88}$. The trigeminal ganglion is also devoid of $\mathrm{BBB}{ }^{89,90}$, suggesting that this feature is a general characteristic of peripheral sensory ganglia.

Stripped of a BBB and thus constitutively exposed to blood-borne factors, spinal ganglia may operate as chemosensors, responding to blood $\mathrm{pH}$ or concentration of metabolites or neuroactive substances ${ }^{91}$, thereby serving as major sites of convergence of neural and humoral signals originating in the body, a role that has typically been associated with more rostral structures in the CNS. Blood-borne chemical signals crossing blood vessel walls and gaining direct access to neuronal somas may bind surface receptors and trigger changes in membrane potential, thereby impacting cellular excitability and action potential propagation, effectively governing the function of spinal ganglia and, consequently, interoceptive processing. 
01/14/19

The three characteristic features of the interoceptive nervous system highlighted abovepredominant use of NST, lack of myelin sheath and presence of gaps in the BBB — are structurally and functionally connected. The dearth of physical barriers such as BBB and myelin effectively exposes the denuded neuronal structures to chemical factors present in the blood stream (in the case of spinal ganglion neuronal cell bodies) and the internal milieu (in the case of unmyelinated axonal trunks of the PNS), providing them with an unparalleled ability to sense circulating molecules and detecting metabolic changes. These denuded, highly sensitized regions of the nervous system constitute an interface between neural and non-neural tissue, akin to the circumventricular organs but peripheral and dispersed throughout the organism rather than central. In all likelihood these regions are more capable of detecting subtler, incipient and localized chemical changes, potentially placing these structures at the very root of interoceptive sensing and, ultimately, of the feelings that allow us to become cognizant of varied states of homeostasis.

The contrasting anatomical and physiological features of synapses and NST (Table 1) suggest a theoretical framework. NST appears ideal to operate the molecular and visceral phenomena expressed in affective experiences ${ }^{15,92,93}$, in full agreement with the previously proposed role for unmyelinated axons in the processing of affect ${ }^{76}$. Whereas movement and perception require precision and gain from speed, the world of affect—moods, and plenty of feelings - can tolerate some vagueness and slowness, perhaps even benefit from them. Affective experiences originate in the graded, modulated transitions from one visceral configuration to another. This would be in keeping with the fact that NST occurs over relatively long timescales — seconds, minutes or even longer-, as opposed to the milli- or even microsecond 
$01 / 14 / 19$

range involved in synaptic transmission ${ }^{94,26}$. Also in support of this notion, the neurotransmitters involved in volume transmission originate mainly in subcortical structures ${ }^{11,22}$, which have been implicated as primary central neural substrates of feeling and subjectivity ${ }^{76}$. Thus synapses may mediate the "what" of neural function (consisting of processes such as sensory perception, skeletal muscle contraction and higher cognition) and NST leads in "how" functions such as affect and arousal ${ }^{15,70,81,95}$. This view has notable implications for the elucidation of the neurophysiology of feelings and, ultimately, for a comprehensive understanding of human mental processes. 
01/14/19

\begin{tabular}{|c|c|c|}
\hline & Synaptic Transmission & Nonsynaptic Transmission \\
\hline Predominant fibers & well myelinated $(A \alpha / A \beta)$ & $\begin{array}{l}\text { poorly myelinated or unmyelinated } \\
(\mathrm{A} \delta / \mathrm{C})\end{array}$ \\
\hline Cell types connected & $\begin{array}{l}\text { neuron-to-neuron, neuron-to- } \\
\text { glia, neuron-to-myocyte }\end{array}$ & any \\
\hline Time scale & Very fast ( $\mu \mathrm{s}-\mathrm{ms})$ & Slow (seconds/minutes/hours) \\
\hline Neural processes supported & $\begin{array}{l}\text { movement, fine perception, } \\
\text { learning, reasoning, } \\
\text { calculation, verbal language }\end{array}$ & $\begin{array}{l}\text { interoception/visceroception, affect } \\
\text { (moods, emotions, feelings) }\end{array}$ \\
\hline $\begin{array}{l}\text { Main areas of the nervous } \\
\text { system }\end{array}$ & $\begin{array}{l}\text { cerebral cortex, main motor } \\
\text { and sensory pathways }\end{array}$ & $\begin{array}{l}\text { limbic system, brain stem, autonomic } \\
\text { nervous system, nociceptive pathways }\end{array}$ \\
\hline Blood-brain barrier & continuous & localized gaps \\
\hline $\begin{array}{l}\text { Main neurotransmitters } \\
\text { and neuromodulators }\end{array}$ & glutamate, GABA & $\begin{array}{l}\text { monoamines (dopamine, noadrenaline, } \\
\text { serotonin), ACh, neuropeptides }\end{array}$ \\
\hline
\end{tabular}

Table 1. General characteristics of synaptic and nonsynaptic transmission modes. 
01/14/19

$\underline{\text { Figure legends }}$

Fig. 1. Synaptic and non-synaptic transmission involve different regulatory mechanisms. A, Synaptic transmission connects neurons via cellular projections (axons/dendrites) and their associated terminal varicosities or synaptic boutons (in red). Establishing a new connection (right, blue neuron) thus involves de novo production of the axon/varicosities/synaptic structures (dashed line). B, Non-synaptic transmission is effected via messenger diffusion. Cells exposed to the messenger can be receptive (black neurons, left) or not (blue neuron, right) depending on the expression of the appropriate surface membrane receptor (in green) recognizing the messenger. A new connection can be established acutely by upregulation of the receptor molecule, without the need for any additional cellular structure.

Fig. 2. Synaptic and non-synaptic transmission interact to achieve signal integration.

Neurotransmitters can 'spill' out of the synaptic cleft and into the surrounding extracellular space (left). Such leaked signaling molecules can activate extrasynaptic membrane receptors (A) or leak into neighboring synapses and modulate intra-synaptic receptors ('intersynaptic spillover', B). Similarly, transmitters released non-synaptically (right) can leak into synaptic compartments and modulate their function (C) or interact with nonsynaptic receptors (D). 
01/14/19

1 Bargmann, C., Beyond the connectome: How neuromodulators shape neural circuits. Bioessays 34, 458-465, (2012).

2 Nickel, M. Vol. 129.1 1-16 (Invertebrate Biology, 2010).

3 Ryan, J. F. \& Chiodin, M. Where is my mind? How sponges and placozoans may have lost neural cell types. Phil. Trans. R. Soc. B 370, 20150059 (2015).

$4 \quad$ Walker, R. \& Holden-Dye, L. Evolutionary aspects of transmitter molecules, their receptors and channels. Parasitology 102, S7-S29 (1991).

5 Walker, R., Brooks, H. \& Holden-Dye, L. Evolution and overview of classical transmitter molecules and their receptors. Parasitology 113, S3-S33 (1996).

6 Wessler, I., Kilbinger, H., Bittinger, F., Unger, R. \& Kirkpatrick, C. J. The non-neuronal cholinergic system in humans: expression, function and pathophysiology. Life sciences 72, 2055-2061 (2003).

7 Moroz, L. L. On the independent origins of complex brains and neurons. Brain, behavior and evolution 74, 177-190 (2009).

8 Ramoino, P. et al. Swimming behavior regulation by GABAB receptors in Paramecium. Experimental cell research 291, 398-405 (2003).

9 Ramoino, P. et al. GABAA receptor subunits identified in Paramecium by immunofluorescence confocal microscopy. FEMS microbiology letters 238, 449-453 (2004).

10 Ramoino, P. et al. GABAB receptor intracellular trafficking after internalization in Paramecium. Microscopy research and technique 68, 290-295 (2005).

11 Haldane, J. La signalisation animale. Annee Biol 58, 89-98 (1954).

12 Nieuwenhuys, R. in Progress in brain research Vol. 125 49-126 (Elsevier, 2000).

13 Fuxe, K., Borroto-Escuela, D. O., Romero-Fernandez, W., Zhang, W.-b. \& Agnati, L. F. Volume transmission and its different forms in the central nervous system. Chinese journal of integrative medicine 19, 323-329 (2013).

14 Taber, K. H. \& Hurley, R. A. Volume transmission in the brain: beyond the synapse. The Journal of neuropsychiatry and clinical neurosciences 26, iv-4 (2014).

15 Agnati, L. F. \& Fuxe, K. Extracellular-vesicle type of volume transmission and tunnelling-nanotube type of wiring transmission add a new dimension to brain neuro-glial networks. Phil. Trans. R. Soc. B 369, 20130505 (2014). 
$01 / 14 / 19$

16 Vizi, E. S., Fekete, A., Karoly, R. \& Mike, A. Non-synaptic receptors and transporters involved in brain functions and targets of drug treatment. Br J Pharmacol 160, 785-809, doi:10.1111/j.1476-5381.2009.00624.x (2010).

17 Bliss, T., Collingridge, G. \& Morris, R. (ROYAL SOC LONDON 6 CARLTON HOUSE TERRACE, LONDON SW1Y 5AG, ENGLAND, 2003).

18 Trueta, C. \& De-Miguel, F. F. Extrasynaptic exocytosis and its mechanisms: a source of molecules mediating volume transmission in the nervous system. Frontiers in physiology 3, 319 (2012).

19 Fuxe, K., Agnati, L. F., Marcoli, M. \& Borroto-Escuela, D. O. Volume Transmission in Central Dopamine and Noradrenaline Neurons and Its Astroglial Targets. Neurochem Res 40, 2600-2614, doi:10.1007/s11064-015-1574-5 (2015).

20 Descarries, L., Gisiger, V. \& Steriade, M. Diffuse transmission by acetylcholine in the CNS. Progress in Neurobiology 53, 603-625 (1997).

21 Umbriaco, D., Watkins, K. C., Descarries, L., Cozzari, C. \& Hartman, B. K. Ultrastructural and morphometric features of the acetylcholine innervation in adult rat parietal cortex: an electron microscopic study in serial sections. Journal of Comparative Neurology 348, 351-373 (1994).

22 Umbriaco, D., Garcia, S., Beaulieu, C. \& Descarries, L. Relational features of acetylcholine, noradrenaline, serotonin and GABA axon terminals in the stratum radiatum of adult rat hippocampus (CA1). Hippocampus 5, 605-620 (1995).

23 Hirase, H., Iwai, Y., Takata, N., Shinohara, Y. \& Mishima, T. Volume transmission signalling via astrocytes. Phil. Trans. R. Soc. B 369, 20130604 (2014).

24 Vizi, E. S. Role of high-affinity receptors and membrane transporters in nonsynaptic communication and drug action in the central nervous system. Pharmacological Reviews 52, 63-90 (2000).

25 Agnati, L. et al. A correlation analysis of the regional distribution of central enkephalin and $\beta$ - endorphin immunoreactive terminals and of opiate receptors in adult and old male rats. Evidence for the existence of two main types of communication in the central nervous system: the volume transmission and the wiring transmission. Acta Physiologica 128, 201-207 (1986).

26 Herkenham, M. Mismatches between neurotransmitter and receptor localizations in brain: observations and implications. Neuroscience 23, 1-38 (1987).

27 Zoli, M., Jansson, A., Syková, E., Agnati, L. F. \& Fuxe, K. Volume transmission in the CNS and its relevance for neuropsychopharmacology. Trends Pharmacol Sci 20, 142-150 (1999). 
01/14/19

28 Fuxe, K. et al. The dopamine D1 receptor-rich main and paracapsular intercalated nerve cell groups of the rat amygdala: relationship to the dopamine innervation. Neuroscience 119, 733-746 (2003).

28 Bjelke, B. et al. Dopaminergic transmission in the rat retina: evidence for volume transmission. Journal of chemical neuroanatomy 12, 37-50 (1996).

30 Schotland, J. L., Shupliakov, O., Grillner, S. \& Brodin, L. Synaptic and nonsynaptic monoaminergic neuron systems in the lamprey spinal cord. Journal of Comparative Neurology 372, 229-244 (1996).

31 Krimer, L. S., Muly III, E. C., Williams, G. V. \& Goldman-Rakic, P. S. Dopaminergic regulation of cerebral cortical microcirculation. Nature neuroscience 1, 286 (1998).

32 Beaudet, A. \& Descarries, L. in Commentaries in the Neurosciences 311-318 (Elsevier, 1980).

33 Fields, R. D. Volume transmission in activity-dependent regulation of myelinating glia. Neurochemistry international 45, 503-509 (2004).

34 Zhang, X., Chen, Y., Wang, C. \& Huang, L.-Y. Neuronal somatic ATP release triggers neuron-satellite glial cell communication in dorsal root ganglia. Proceedings of the National Academy of Sciences 104, 9864-9869 (2007).

35 Szapiro, G. \& Barbour, B. Parasynaptic signalling by fast neurotransmitters: the cerebellar cortex. Neuroscience 162, 644-655 (2009).

36 Vizi, E. S., Kiss, J. P. \& Lendvai, B. Nonsynaptic communication in the central nervous system. Neurochemistry international 45, 443-451 (2004).

37 Veening, J. G., Gerrits, P. O. \& Barendregt, H. P. Volume transmission of beta-endorphin via the cerebrospinal fluid; a review. Fluids and Barriers of the CNS 9, 16 (2012).

38 Kiss, J. P. \& Vizi, E. S. Nitric oxide: a novel link between synaptic and nonsynaptic transmission. Trends in neurosciences 24, 211-215 (2001).

39 Ryter, S. W., Otterbein, L. E., Morse, D. \& Choi, A. M. in Oxygen/Nitrogen Radicals: Cell Injury and Disease 249-263 (Springer, 2002).

40 Agnati, L. \& Fuxe, K. in Progress in brain research Vol. 125 3-19 (Elsevier, 2000).

41 Banke, T. G., Dravid, S. M. \& Traynelis, S. F. Protons trap NR1/NR2B NMDA receptors in a nonconducting state. Journal of Neuroscience 25, 42-51 (2005).

42 Nemeth, E. F. in Principles of Bone Biology (Second Edition) 1339-1359 (Elsevier, 2002). 
$01 / 14 / 19$

43 Konsman, J. P., Parnet, P. \& Dantzer, R. Cytokine-induced sickness behaviour: mechanisms and implications. Trends in neurosciences 25, 154-159 (2002).

44 Lang, P. M., Tracey, D. J., Irnich, D., Sippel, W. \& Grafe, P. Activation of adenosine and P2Y receptors by ATP in human peripheral nerve. Naunyn Schmiedebergs Arch Pharmacol 366, 449-457, doi:10.1007/s00210-002-0624-0 (2002).

45 Lang, P. M., Moalem-Taylor, G., Tracey, D. J., Bostock, H. \& Grafe, P. Activitydependent modulation of axonal excitability in unmyelinated peripheral rat nerve fibers by the 5-HT(3) serotonin receptor. J Neurophysiol 96, 2963-2971, doi:10.1152/jn.00716.2006 (2006).

46 Lang, P. M. \& Grafe, P. Chemosensitivity of unmyelinated axons in isolated human gastric vagus nerve. 136, 100-104, doi:10.1016/j.autneu.2007.05.001 (2007).

47 Irnich, D., Tracey, D. J., Polten, J., Burgstahler, R. \& Grafe, P. ATP stimulates peripheral axons in human, rat and mouse--differential involvement of $\mathrm{A}(2 \mathrm{~B})$ adenosine and $\mathrm{P} 2 \mathrm{X}$ purinergic receptors. Neuroscience 110, 123-129 (2002).

48 Cockayne, D. A. et al. Urinary bladder hyporeflexia and reduced pain-related behaviour in P2X3-deficient mice. Nature 407, 1011-1015, doi:10.1038/35039519 (2000).

49 Amir, R. \& Devor, M. Functional cross-excitation between afferent A-and C-neurons in dorsal root ganglia. Neuroscience 95, 189-195 (1999).

50 Oh, E. J. \& Weinreich, D. Chemical communication between vagal afferent somata in nodose Ganglia of the rat and the Guinea pig in vitro. Journal of Neurophysiology 87, 2801-2807 (2002).

51 Amir, R. \& Devor, M. Chemically mediated cross-excitation in rat dorsal root ganglia. Journal of Neuroscience 16, 4733-4741 (1996).

52 Omoto, K. et al. Cross-excitation in peripheral sensory ganglia associated with pain transmission. Toxins 7, 2906-2917 (2015).

53 Du, X. et al. Control of somatic membrane potential in nociceptive neurons and its implications for peripheral nociceptive transmission. PAIN® 155, 2306-2322 (2014).

54 Burnstock, G. Non-synaptic transmission at autonomic neuroeffector junctions. Neurochemistry international 52, 14-25 (2008).

55 Franklin, I. K. \& Wollheim, C. B. GABA in the endocrine pancreas: its putative role as an islet cell paracrine-signalling molecule. The Journal of general physiology 123, 185190 (2004).

56 Rodriguez-Diaz, R. et al. Alpha cells secrete acetylcholine as a non-neuronal paracrine signal priming beta cell function in humans. Nature medicine 17, 888 (2011). 
$01 / 14 / 19$

57 de Almeida, J. L. \& Saldanha, C. Nonneuronal cholinergic system in human erythrocytes: biological role and clinical relevance. Journal of Membrane Biology 234, 227-234 (2010).

58 Mayerhofer, A., Dimitrijevic, N. \& Kunz, L. The expression and biological role of the non-neuronal cholinergic system in the ovary. Life sciences 72, 2039-2045 (2003).

59 Agnati, L. et al. Energy gradients for the homeostatic control of brain ECF composition and for VT signal migration: introduction of the tide hypothesis. Journal of Neural Transmission 112, 45-63 (2005).

60 Fuxe, K. et al. Dynamics of volume transmission in the brain. Focus on catecholamine and opioid peptide communication and the role of uncoupling protein 2. Journal of neural transmission 112, 65-76 (2005).

61 Fuxe, K. \& Borroto-Escuela, D. O. Volume transmission and receptor-receptor interactions in heteroreceptor complexes: understanding the role of new concepts for brain communication. Neural regeneration research 11, 1220 (2016).

62 Paspalas, C. D. \& Goldman-Rakic, P. S. Microdomains for dopamine volume neurotransmission in primate prefrontal cortex. Journal of Neuroscience 24, 5292-5300 (2004).

63 Smiley, J. F., Levey, A. I., Ciliax, B. J. \& Goldman-Rakic, P. S. D1 dopamine receptor immunoreactivity in human and monkey cerebral cortex: predominant and extrasynaptic localization in dendritic spines. Proceedings of the National Academy of Sciences 91, 5720-5724 (1994).

64 Fuxe, K. et al. On the role of volume transmission and receptor-receptor interactions in social behaviour: focus on central catecholamine and oxytocin neurons. Brain research 1476, 119-131 (2012).

65 Savtchenko, L. P. \& Rusakov, D. A. The optimal height of the synaptic cleft. Proceedings of the National Academy of Sciences 104, 1823-1828 (2007).

66 van den Pol, A. N. Neuropeptide transmission in brain circuits. Neuron 76, 98-115 (2012).

67 Nicholson, C. \& Syková, E. Extracellular space structure revealed by diffusion analysis. Trends in neurosciences 21, 207-215 (1998).

68 Fuxe, K. et al. The discovery of central monoamine neurons gave volume transmission to the wired brain. Prog Neurobiol 90, 82-100, doi:10.1016/j.pneurobio.2009.10.012 (2010).

69 Zoli, M. et al. The emergence of the volume transmission concept1. Brain Research Reviews 26, 136-147 (1998). 
$01 / 14 / 19$

70 Bach-y-Rita, P. \& Aiello, G. L. Nerve length and volume in synaptic vs diffusion neurotransmission: a model. NeuroReport 7, 1502-1504 (1996).

71 Castañeda-Hernández, G. C. \& Bach-y-Rita, P. Volume transmission and pain perception. The Scientific World Journal 3, 677-683 (2003).

72 Aiello, G. \& Bach-y-Rita, P. Hebbian brain cell-assemblies: nonsynaptic neurotransmission, space and energy considerations. Intelligent engineering systems through artificial neural networks 11, 441-447 (2001).

73 Fuxe, K. et al. Moonlighting proteins and protein-protein interactions as neurotherapeutic targets in the $\mathrm{G}$ protein-coupled receptor field. Neuropsychopharmacology 39, 131 (2014).

74 Borroto-Escuela, D. O. et al. On the G-protein-coupled receptor heteromers and their allosteric receptor-receptor interactions in the central nervous system: focus on their role in pain modulation. Evidence-Based Complementary and Alternative Medicine $\mathbf{2 0 1 3}$ (2013).

75 Purves, D. Neuroscience. Sixth edition. edn, (Oxford University Press, 2018).

76 Calabresi, P., Ghiglieri, V., Mazzocchetti, P., Corbelli, I. \& Picconi, B. Levodopainduced plasticity: a double-edged sword in Parkinson's disease? Phil. Trans. R. Soc. B 370, 20140184 (2015).

77 Damasio, A. \& Carvalho, G. The nature of feelings: evolutionary and neurobiological origins. Nature Reviews Neuroscience 14, 143-152, doi:doi:10.1038/nrn3403 (2013).

78 Bokil, H., Laaris, N., Blinder, K., Ennis, M. \& Keller, A. Ephaptic interactions in the mammalian olfactory system. J Neurosci 21, RC173 (2001).

79 Moalem, G., Grafe, P. \& Tracey, D. Chemical mediators enhance the excitability of unmyelinated sensory axons in normal and injured peripheral nerve of the rat.

Neuroscience 134, 1399-1411 (2005).

80 Nieuwenhuys, R., Veening, J. \& Van Domburg, P. Core and paracores; some new chemoarchitectural entities in the mammalian neuraxis. Acta Morphologica NeerlandoScandinavica 26, 131-163 (1988).

81 Nieuwenhuys, R. Chemoarchitecture of the Brain. (Springer Science \& Business Media, 2012).

82 Nieuwenhuys, R. in Progress in brain research Vol. $107 \quad$ 551-580 (Elsevier, 1996).

83 Damasio, A. R. The strange order of things: life, feeling, and the making of the cultures. (Pantheon Books, 2018). 
$01 / 14 / 19$

84 Syková, E. \& Chvátal, A. Glial cells and volume transmission in the CNS. Neurochemistry international 36, 397-409 (2000).

85 Sykova, E. Extrasynaptic volume transmission and diffusion parameters of the extracellular space. Neuroscience 129, 861-876 (2004).

86 Sykova, E. Glia and volume transmission during physiological and pathological states. Journal of Neural Transmission 112, 137-147 (2005).

87 Jimenez-Andrade, J. M. et al. Vascularization of the dorsal root ganglia and peripheral nerve of the mouse: implications for chemical-induced peripheral sensory neuropathies. Molecular pain 4, 10 (2008).

88 Jacobs, J. M. Vascular permeability and neurotoxicity. Environmental health perspectives 26, 107 (1978).

89 Hirakawa, H. et al. Regional differences in blood-nerve barrier function and tightjunction protein expression within the rat dorsal root ganglion. Neuroreport 15, 405-408 (2004).

90 Lundblad, C., Haanes, K. A., Grände, G. \& Edvinsson, L. Experimental inflammation following dural application of complete Freund's adjuvant or inflammatory soup does not alter brain and trigeminal microvascular passage. The journal of headache and pain 16, 91 (2015).

91 Eftekhari, S. et al. Localization of CGRP, CGRP receptor, PACAP and glutamate in trigeminal ganglion. Relation to the blood-brain barrier. Brain research 1600, 93-109 (2015).

92 Devor, M. Unexplained peculiarities of the dorsal root ganglion. Pain 82, S27-S35 (1999).

93 Agnati, L. F. et al. Neuronal correlates to consciousness. The "Hall of Mirrors" metaphor describing consciousness as an epiphenomenon of multiple dynamic mosaics of cortical functional modules. Brain Research 1476, 3-21 (2012).

94 Locks, J. T. New Research on Consciousness. (Nova Publishers, 2007).

95 Borroto-Escuela, D. O. et al. The role of transmitter diffusion and flow versus extracellular vesicles in volume transmission in the brain neural-glial networks. Philos Trans R Soc Lond B Biol Sci 370, doi:10.1098/rstb.2014.0183 (2015).

96 Bach-y-Rita, P. Thoughts on the role of volume transmission in normal and abnormal mass sustained functions. Volume Transmission in the Brain, 489-496 (1991). 
01/14/19
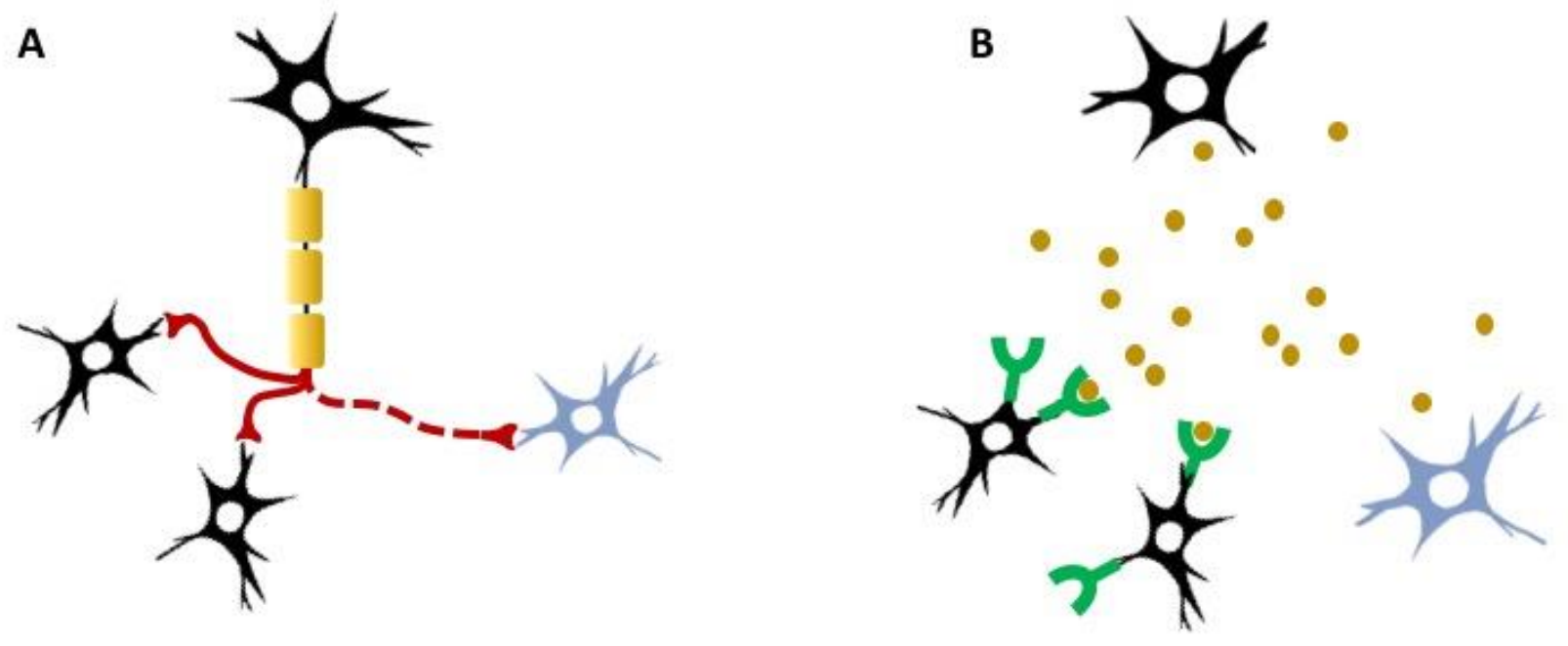

Fig. 1 
01/14/19

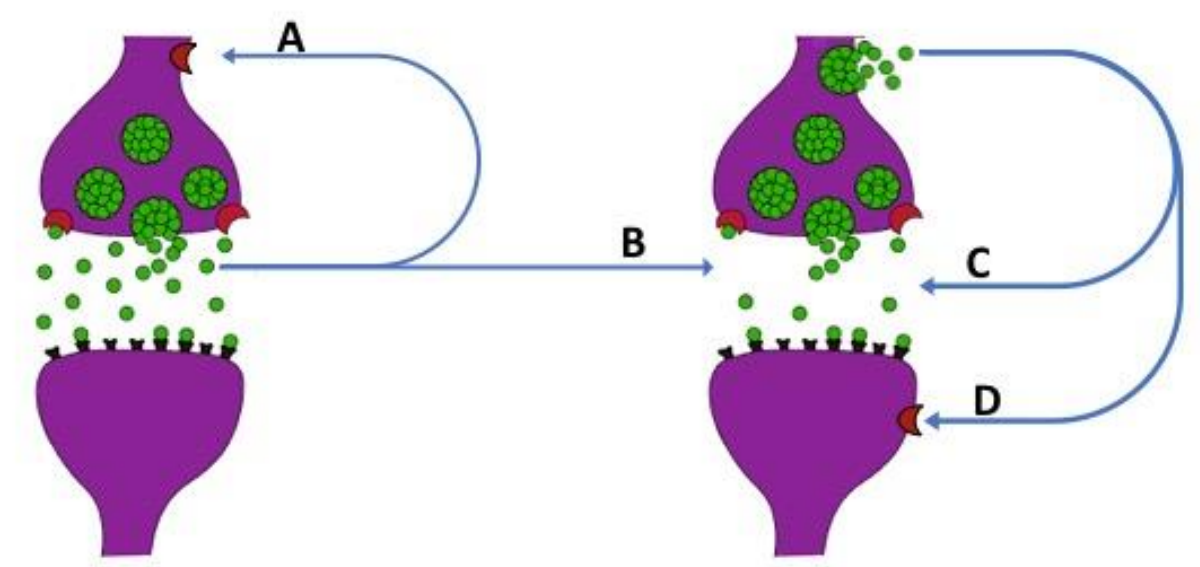

Fig. 2 